\title{
Vescicole extracellulari e metabolismo osseo: ruolo e possibili implicazioni cliniche
}

\author{
Simone Bianciardi ${ }^{1}$. Daniela Merlotti ${ }^{1} \cdot$ Maria Materozzi $^{1,2}$
}

Accettato: 29 maggio 2020 / Pubblicato online: 17 febbraio 2021

(c) The Author(s) 2021

Sommario Le vescicole extracellulari (EV) sono secrete da vari tipi cellulari e veicolano cargo specifici destinati alle cellule target, mediando così effetti paracrini in meccanismi fisiologici e patologici. Negli ultimi anni lo studio delle EV si è affermato in moltissimi ambiti, compreso il mondo del metabolismo osseo. In questa rassegna riportiamo i più recenti e promettenti studi sul ruolo biologico delle EV nel tessuto osseo e il loro potenziale utilizzo in strategie terapeutiche.

Parole chiave Vescicole Esosomi - Rigenerazione ·

Osteoporosi $\cdot$ Metastasi $\cdot$ Metabolismo osseo

\section{Introduzione}

Le vescicole extracellulari (EV) sono piccole vescicole rivestite da un doppio strato fosfolipidico, prodotte da molte tipologie cellulari con lo scopo di veicolare il proprio contenuto (cargo) alle cellule bersaglio. Hanno dimensioni che possono variare da $20 \mathrm{~nm}$ a $2 \mu \mathrm{m}$, fino ad arrivare oltre $10 \mu \mathrm{m}$ nel caso degli oncosomi. La loro presenza è confermata in quasi tutti i liquidi e i tessuti biologici compreso l'osso, dove contribuiscono a regolare il metabolismo e le comunicazioni

Proposto da Vito Guarnieri.

Informazioni Supplementari La versione online contiene materiale supplementare disponibile su

https://doi.org/10.1007/s40619-021-00817-4.

S. Bianciardi

simo_1988@libero.it

1 Dipartimento di Scienze Mediche, Chirurgiche e Neuroscienze, Università degli studi di Siena, Siena, Italia

2 Divisione di Genetica e Biologia Cellulare, Istituto Scientifico San Raffaele, Milano, Italia intercellulari sia in condizioni fisiologiche che patologiche. Il numero, le dimensioni e il contenuto delle EV può variare in base alle cellule di origine, alla presenza o meno di patologie e al microambiente in cui sono inserite $[1,2]$.

\section{EV: classificazione, biogenesi e funzioni}

Sulla base delle dimensioni e del meccanismo di biogenesi le EV possono essere distinte in esosomi, microvescicole (MV) e corpi apoptotici. Gli esosomi sono un gruppo eterogeneo di vescicole con dimensioni che variano da 40 a $160 \mathrm{~nm}$ la cui formazione può essere divisa in tre passaggi: 1) invaginazione della membrana plasmatica a formare l'endosoma; 2) invaginazione della membrana endosomica a formare un multi vesicular body (MVB) con all'interno le vescicole intraluminali (gli esosomi veri e propri); e 3) fusione dei MVB con la membrana cellulare e conseguente rilascio degli esosomi all'esterno (Fig. 1) [3]. Il processo può essere dipendente dal meccanismo endosomal sorting complex required for transport (ESCRT) o indipendente da esso in seguito alla formazione della ceramide o all'intervento di proteine della famiglia delle tetraspanine. Entrambi i meccanismi cooperano e il loro contributo varia in funzione del contenuto e del tipo cellulare. Le MV hanno dimensioni comprese fra $50 \mathrm{~nm}$ e $1 \mu \mathrm{m}$ e si originano direttamente dalla membrana plasmatica mediante gemmazione verso l'esterno. Questo processo richiede un cambiamento dell'assetto lipidico di membrana (le MV sono ricche di fosfatidilserina), della composizione proteica e della concentrazione di $\mathrm{Ca}^{2+}$. I corpi apoptotici si formano in seguito alla frammentazione della cellula secondaria al processo di apoptosi e hanno una dimensione compresa fra 0,5 e $4 \mu \mathrm{m}[1,2]$.

Una volta nell' ambiente extracellulare, le EV possono indurre una risposta nelle cellule bersaglio in due modi: atti- 
Fig. 1 Biogenesi delle tre principali EV. MVB, Multi Vesicular Body; $I L V$, vescicole intraluminali. Rappresentazione delle tre vie che portano alla formazione delle varie EV: via endocitotica, apoptosi e gemmazione della membrana

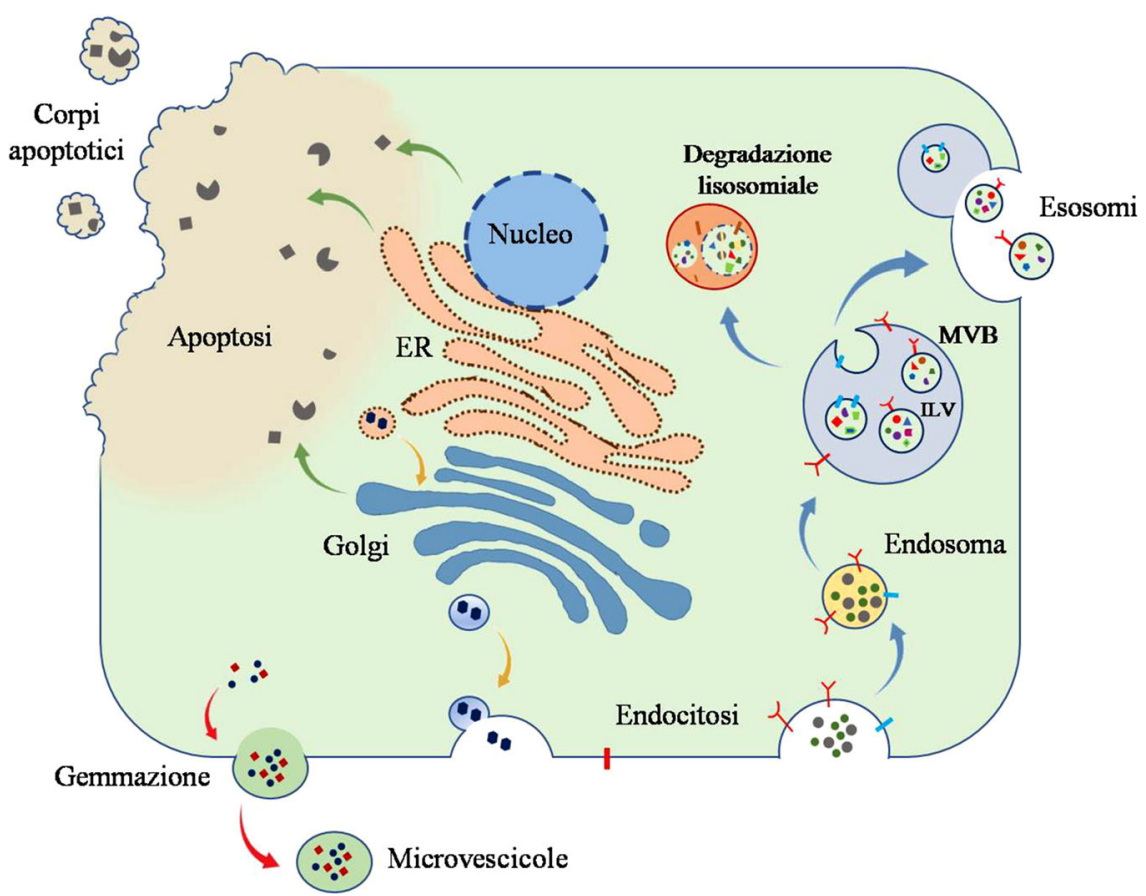

vando recettori di membrana oppure rilasciando nel citoplasma il proprio cargo che può contenere: proteine (es. fattori di trascrizione), acidi nucleici (es. mRNA e miRNA) e lipidi. Le EV sono, quindi, importanti mediatori inter-cellulari e sono coinvolti in una moltitudine di processi, sia fisiologici (coagulazione, sorveglianza immunitaria, ecc.) che patologici (tumorigenesi, formazione di metastasi, ecc.) [2].

\section{EV nel metabolismo osseo}

Anche nel tessuto osseo troviamo un microambiente arricchito di EV secrete da ogni tipo cellulare presente (compreso endotelio, cellule dell'immunità, ecc.). In questa rassegna ci concentreremo sul ruolo delle EV secrete da osteoblasti $(\mathrm{OB})$, osteoclasti (OC) e osteociti, i cui più recenti studi sono riassunti in Tabella 1.

Le cellule stromali/staminali del midollo osseo (BMSC) hanno un ruolo fondamentale nel differenziamento osteoblastico non solo come precursori, ma anche come mediatori paracrini attraverso la secrezione di EV regolatorie che, endocitate dagli OB, promuovono l'osteogenesi. Le molecole principalmente coinvolte sono i miRNA, come per esempio $\mathrm{i}$ miR-196a, miR-27a, e miR-206 che, una volta trasferiti agli $\mathrm{OB}$, inducono l'espressione di geni chiave, quali $R U N X 2 \mathrm{e}$ $A L P$, promuovendo il differenziamento e l'osteogenesi [4]. Un recente studio ha dimostrato un potente effetto regolatorio anche da parte di un lncRNA, il MALAT1, che si trova in grandi quantità negli esosomi estratti da BMSC umane primarie. In co-coltura con OB umani (linea hFOB1.19),
MALAT1 rilasciato negli esosomi va a inibire il miR-34c, promuovendo il differenziamento tramite l'upregolazione di SATB2. Questo meccanismo ha dimostrato efficacia anche in vivo in topi con osteoporosi (OP) secondaria a ovariectomia (OVX) [5]. Una volta differenziati, gli OB sono grandi produttori di EV funzionalmente attive nella regolazione del processo di osteogenesi, tale produzione può inoltre cambiare nei vari stadi del differenziamento. Nei modelli murini è nella fase terminale che gli OB (MC3T3-E1) rilasciano MV che promuovono fortemente il differenziamento nelle BM$\mathrm{SC}$ primarie e questo effetto selettivo è mantenuto anche in vivo, dove attenuano l'OP in topi OVX [6]. Le EV rappresentano inoltre un'importante mezzo di comunicazione OBOC con effetti sia pro- che anti-osteoclastogenici. In modelli murini le EV di OB sono ricche di RANKL che, attivando RANK sulla superficie dei monociti (BMM), precursori osteoclastici, ne promuovono il differenziamento (OCgenesi) e la sopravvivenza [7]. Al contrario, queste possono inibire la formazione degli OC tramite il miR-125b che, rilasciato nella matrice, viene poi captato dagli OC e reprime PRDM, noto fattore trascrizionale pro-osteoclastogenico, target di NFATc1 [8]. Anche gli OC sfruttano EV per regolare il rimodellamento osseo, agendo sia sugli OB che sul proprio processo di formazione. Un recente lavoro ha infatti identificato un potente meccanismo regolatorio da parte del miR$23 \mathrm{a}-5 \mathrm{p}$, contenuto in grandi quantità negli esosomi osteoclastici, che in coltura è in grado di inibire $R U N X 2$, reprimendo l'osteoblastogenesi [9]. Gli OC rilasciano inoltre EV con numerosi miRNA e RANK stesso che, entrando in competizione con il suo ligando (solubile o sulle membrane degli 
Tabella 1 Ruolo delle EV nel rimodellamento osseo e meccanismi molecolari coinvolti. $B M S C$, cellule stromali/staminali del midollo osseo; $O B$, osteoblasti; $O C$, osteoclasti; $B M M$, monociti

\begin{tabular}{|c|c|c|c|c|}
\hline $\begin{array}{l}\text { Cellula di } \\
\text { origine }\end{array}$ & $\begin{array}{l}\text { Cargo } \\
\text { delle EV }\end{array}$ & $\begin{array}{l}\text { Cellula } \\
\text { target }\end{array}$ & $\begin{array}{l}\text { Effetto } \\
\text { biologico }\end{array}$ & $\begin{array}{l}\text { Meccanismo e } \\
\text { molecole coinvolte }\end{array}$ \\
\hline BMSC & miR-196a, miR-27a e miR-206 & OB & $\uparrow$ Osteogenesi & $\uparrow$ RUNx2, ALP, OCN, OPN \\
\hline BMSC & MALAT1 & OB & $\uparrow$ Osteogenesi & Asse miR-34c/SATB2 \\
\hline BMSC & $\operatorname{miR}-122-5 p$ & OB & $\uparrow$ Osteogenesi & $\downarrow$ SPRY2 \\
\hline BMSC & non specificato & $\mathrm{OB}$ & $\uparrow$ Osteogenesi & $\uparrow$ GLUT3 \\
\hline $\mathrm{OB}$ & $\begin{array}{l}\operatorname{miR}-667-3 p, \operatorname{miR}-6769 b-5 p \\
\text { miR-7044-5p, miR-7668-3p e } \\
\text { miR-874-3p }\end{array}$ & BMSC & $\uparrow$ Osteogenesi & $\downarrow$ Axin $1, \mathrm{Wnt} / \beta$-catenin \\
\hline OB & $\begin{array}{l}\text { Proteine della matrice } \\
\text { (TenascinC, fibronectina, } \\
\text { collagene, TRIP1) }\end{array}$ & $\begin{array}{l}\text { Matrice; } \\
\text { BMSC }\end{array}$ & $\uparrow$ Osteogenesi & - \\
\hline $\mathrm{OB}$ & RANKL & BMM & $\uparrow$ OCgenesi & $\uparrow$ Asse RANKL/RANK \\
\hline OB & $\operatorname{miR}-125 b$ & $\mathrm{OC}$ & $\downarrow$ OCgenesi & $\downarrow$ PRDM1 \\
\hline $\mathrm{OB}$ & MMP2 & Endotelio & $\uparrow$ Angiogenesi & $\uparrow$ VEGF/Erk1/2 \\
\hline BMM & RNA, miRNA (non specificati) & BMSC & $\uparrow$ Osteogenesi & $\uparrow \mathrm{RUNX} 2, \mathrm{BMP} 2$ \\
\hline $\mathrm{OC}$ & RANK & BMM & $\downarrow$ OCgenesi & $\begin{array}{l}\text { blocco di RANKL e } \\
\text { sRANKL }\end{array}$ \\
\hline $\mathrm{OC}$ & $\operatorname{miR}-214-3 p$ & OB & $\downarrow$ Osteogenesi & $\downarrow$ ATF4 \\
\hline $\mathrm{OC}$ & $\operatorname{miR}-214-3 p$ & $\mathrm{OC}$ & $\uparrow$ OCgenesi & $\uparrow \mathrm{Akt} / \mathrm{PI} 3 \mathrm{~K}$ \\
\hline $\mathrm{OC}$ & RANK (corpi apoptotici) & OB & $\uparrow$ Osteogenesi & $\begin{array}{l}\uparrow \mathrm{Akt} / \mathrm{mTOR} \text { (RANKL } \\
\text { reverse signaling) }\end{array}$ \\
\hline $\mathrm{OC}$ & $\operatorname{miR}-23 a-5 p$ & OB & $\downarrow$ Osteogenesi & $\downarrow$ RUNX2 \\
\hline Osteociti & $\operatorname{miR}-218$ & OB & $\downarrow$ Osteogenesi & $\downarrow$ SOST, TCF7 \\
\hline Osteociti & Sclerostina, RANKL, OPG & OB & $\uparrow$ Osteogenesi & \\
\hline
\end{tabular}

OB), riduce la sua biodisponibilità e, pertanto, attenua l'OCgenesi che, in questo modo, viene strettamente controllata tramite feedback negativi. L'asse RANKL/RANK potrebbe anche essere sfruttato per veicolare molecole agli OB [10]. Un altro tipo di $\mathrm{EV}$ ha un ruolo nel rimodellamento osseo, in particolare nella comunicazione OC-OB: i corpi apoptotici. Condotti alla morte, per esempio con bisfosfonati, gli OC rilasciano corpi apoptotici ricchi di RANK che, legandosi a RANKL sulla membrana degli $\mathrm{OB}$, induce un reverse signaling tramite il pathway Akt/mTOR, stimolandone la sopravvivenza e il differenziamento. Tali corpi apoptotici hanno anche dimostrato in vitro un potenziale osteogenico maggiore rispetto alle altre EV secrete dagli osteoclasti [11].

Sebbene OB e OC rimangano per il momento al centro dei più recenti studi, anche gli osteociti, importanti regolatori del rimodellamento osseo, rilasciano EV in seguito a stimoli biochimici e meccanici (loading) [4]. Uno dei meccanismi d'azione recentemente identificato vede come protagonista il miR-218, che attenua l'osteogenesi andando a bloccare negli OB la sintesi di proteine chiave del pathway di Wnt (come per esempio SOST e TCF7) [12].

\section{Ruolo delle EV nelle metastasi ossee}

Lo scheletro è uno degli organi più frequentemente coinvolto nella formazione di metastasi $(80 \%$ delle quali interessa le aree a elevata vascolarizzazione come vertebre, anche e costole), preceduto solamente dal polmone e dal fegato. I tumori che metastatizzano nel tessuto osseo con maggiore frequenza sono quelli di mammella, prostata e polmone, con un'incidenza rispettiva del $70 \%, 80 \%$ e $40 \%$ [13]. In condizioni fisiologiche il riassorbimento della matrice ossea da parte degli OC è finemente bilanciato dalla formazione di nuovo tessuto ad opera degli OB. In presenza di metastasi questo equilibrio viene a mancare e si possono creare due scenari opposti: una lesione osteolitica, nella quale prevale l'attività riassorbitiva degli $\mathrm{OC} \mathrm{o}$ una lesione osteosclerotica, nella quale prevale la formazione di nuovo tessuto [14]. Oltre all'importante ruolo svolto dalle EV nella comunicazione intercellulare, recentemente è stato chiarito anche il loro coinvolgimento nella metastatizzazione; essi, infatti, possono da un lato aumentare il potere metastatizzante delle cellule tumorali e, dall'altro, promuovere la formazione di una "nicchia premetastatica" nel tessuto bersaglio attraverso un'azione paracrina, favorendo l'insorgenza della metastasi vera e propria $[15,16]$. 
Tabella 2 Ruolo delle EV nella formazione delle metastasi ossee e meccanismi molecolari coinvolti

\begin{tabular}{|c|c|c|c|c|c|}
\hline $\begin{array}{l}\text { Tumori } \\
\text { di origine }\end{array}$ & $\begin{array}{l}\text { Linea } \\
\text { cellulare }\end{array}$ & EV & Cargo & Effetto biologico & $\begin{array}{l}\text { Meccanismo } \\
\text { molecolare }\end{array}$ \\
\hline Prostata & TRAMP-C1 & Esosomi & - & $\downarrow$ OCgenesi & - \\
\hline Prostata & $\mathrm{LNCaP}$ & Esosomi & $\operatorname{miR}-375$ & $\begin{array}{l}\uparrow \text { Osteoblastogenesi } \\
\uparrow \text { OB attività }\end{array}$ & - \\
\hline Prostata & - & Esosomi & miR-940 & $\begin{array}{l}\uparrow \text { Osteoblastogenesi } \\
\uparrow \text { Osteoblastogenesi }\end{array}$ & $\begin{array}{l}\uparrow \text { ARHGAP1 e } \\
\text { FAM134A } \\
\downarrow \text { Pathway } \\
\text { RhoA/ROCK }\end{array}$ \\
\hline Prostata & PC3-DU145 & MV & $\begin{array}{l}\text { Protoncogene } \\
\text { Ets1 }\end{array}$ & $\uparrow$ Osteoblastogenesi & - \\
\hline Polmone & $\begin{array}{l}\text { CRL-2868 } \\
\text { A549 }\end{array}$ & Esosomi & Anfiregulina & $\uparrow$ OCgenesi & $\uparrow$ Pathway EGFR \\
\hline Polmone & A549 & $\begin{array}{l}\text { Vescicole } \\
\text { Simil } \\
\text { esosomi }\end{array}$ & miR-192 & $\begin{array}{l}\downarrow \text { Formazione delle } \\
\text { metastasi }\end{array}$ & $\begin{array}{l}\downarrow \text { Angiogenesi } \\
\downarrow \text { Colonizzazione }\end{array}$ \\
\hline
\end{tabular}

Tali meccanismi sono oggetto di studio anche nel tessuto osseo dove EV tumorali possono alterare il comportamento delle cellule residenti in modo da creare un microambiente ideale per la formazione di lesioni secondarie. Yang e collaboratori hanno dimostrato, per esempio, che esosomi rilasciati da cellule di cancro prostatico (PC-3) inibiscono il differenziamento degli $\mathrm{OC}$ andando a bloccare il pathway di NF-kB attraverso il miR-214 [14]. Oltre a inibire il riassorbimento viene indotta l'osteogenesi, promuovendo il differenziamento e l'attività degli $\mathrm{OB}$. In questo contesto giocano un ruolo chiave i miRNA presenti nelle EV; Probert e colleghi hanno infatti dimostrato non solo che gli EV dalle PC-3 possono aumentare la vitalità degli $\mathrm{OB}$, ma anche che questo effetto viene perso silenziando Dicer (una proteina fondamentale per la genesi dei miRNA) [17]. Il miR-141-3p è uno dei principali mediatori di tale effetto. È arricchito negli esosomi delle cellule MDA PCa 2c (linea tumorale prostatica che forma metastasi ossee) e svolge la propria azione pro-osteoblastica bloccando DLC1, un inibitore del pathway di p38 MAPK. Parallelamente miR-141-3p aumenta il rapporto OPG/RANKL, inibendo così l'OCgenesi [18].

Per quanto riguarda le metastasi di natura osteolitica (tipiche del tumore al polmone), le EV svolgono un ruolo proosteoclastogenico. Prendendo come modello la linea cellulare polmonare A549 Zhen et al. hanno dimostrato come queste non solo iper-esprimano il miR-21, ma siano anche in grado di veicolarlo ai precursori degli OC mediante esosomi, promuovendone così il differenziamento attraverso l'inibizione di Pdcd4 [19]. Infine, Loftus e colleghi hanno provato che gli EV rilasciati dalle cellule tumorali del cancro al seno vanno ad agire direttamente sugli $\mathrm{OB}$ riducendone il numero e l'attività, e sugli $\mathrm{OC}$, promuovendone il differenziamento in maniera RANKL-indipendente. Parallelamente, incrementano l'angiogenesi e "istruiscono" gli OB al fine di aumentarne la produzione e il rilascio di fattori pro- osteoclastogenici, pro-infiammatori e pro-angiogenici [20]. Altri meccanismi di azione delle EV nei tumori sono riassunti nella Tabella 2. Nonostante molti meccanismi molecolari siano ancora da chiarire, i risultati finora ottenuti rendono questo campo di studio molto promettente sia per meglio comprendere il meccanismo mediante il quale le metastasi si originano, sia per la possibile individuazione di nuovi bersagli terapeutici.

\section{Ruolo delle EV nella perdita di massa ossea legata all'invecchiamento}

Anche con l'invecchiamento l'equilibrio fisiologico fra riassorbimento e osteogenesi tende ad essere meno efficiente, facendo sì che il primo prevarichi sull'osteogenesi portando alla riduzione della massa osseo e all' aumento del rischio di fratture, condizione tipica dell'OP senile [21].

Numerosi studi hanno dimostrato che le cellule senescenti subiscono un'alterazione del secretoma che prende il nome di senescence-associated secretory phenotype (SASP). Questi fattori rilasciati nel comparto extracellulare possono sfavorire la riparazione e la rigenerazione dei tessuti. Anche le EV si modificano con la senescenza e possono causare alterazioni nel crosstalk fra le popolazioni cellulari del tessuto osseo [22]. Fra i componenti del SASP, i miRNA rilasciati all'interno delle EV giocano un ruolo molto importante nel processo di rimodellamento osseo età-dipendente. Le BMSC di ratti anziani, infatti, esprimono alti livelli di miR-31a$5 \mathrm{p}$ che, rilasciato tramite esosomi, promuove la senescenza bloccando E2F2, inibisce il differenziamento osteogenico e parallelamente promuove OCgenesi e riassorbimento, agendo sulla via di RhoA [21]. È interessante notare come anche cellule non ossee, ma strettamente connesse al microambiente scheletrico, in condizioni di senescenza possa- 
no rilasciare EV in grado di compromettere l'osteogenesi. È il caso delle cellule endoteliali e di quelle muscoloscheletriche, che rispettivamente producono e rilasciano alti livelli di miR-31 e miR-34, due miRNA che riducono il differenziamento osteogenico e la vitalità delle cellule staminali mesenchimali $[22,23]$. Simili effetti sono osservati anche nell'uomo, dove Li e collaboratori hanno evidenziato alti livelli di miR-214-3p nel siero e negli OC di donne anziane con fratture. Tale miRNA, rilasciato via esosomi, viene internalizzato dagli $\mathrm{OB}$ dove ne riduce drasticamente l'attività causando la perdita di massa ossea [24]. Nel complesso, ottenere una migliore comprensione dei meccanismi legati all'insorgenza dell'OP senile può aiutare a combattere in maniera più efficace una patologia che, con il costante aumento dell'età generale della popolazione, sta diventando sempre più preponderante nella nostra società.

\section{Possibili applicazioni delle EV}

Uno degli aspetti più affascinanti delle EV è senz'altro il loro potenziale clinico: esse potrebbero infatti rappresentare nuove strategie terapeutiche, sia sfruttando il loro cargo naturale che modificandolo per veicolare farmaci. È facile pensare a un loro utilizzo per veicolare chemioterapici in modo selettivo sul tumore, risparmiando i gravi effetti indesiderati sugli altri tessuti, grazie ai recettori di cui sono dotate (es. il sistema EphrinA2/EphA2). Si sono anche dimostrate un efficiente metodo di drug delivery, come nel caso di zoledronato e dasatinib [7]. Sempre più studi hanno investigato un possibile utilizzo terapeutico in malattie ossee e nella rigenerazione post-frattura, principalmente sfruttando le EV di cellule mesenchimali (MSC). Queste sono, per esempio, in grado di stimolare la crescita dei condrociti in modelli di osteogenesi imperfetta e di aumentare la riparazione del tessuto dopo una frattura (tramite il coinvolgimento dei miR21, miR-4532 e miR-125b-5p) [25]. E ancora, le EV di MSC derivanti da cordone ombelicale umano o da tessuto adiposo hanno tutte dimostrato grande efficacia in studi animali nel promuovere la rigenerazione o l'osteogenesi in modelli di OP, osteonecrosi o frattura [26, 27]. Le EV, quindi, potrebbero rappresentare un'alternativa al trapianto di cellule staminali per la rigenerazione ossea. Sebbene ad oggi i dati disponibili derivino da studi animali, sappiamo che anche gli esosomi umani hanno questi benefici: le EV estratte direttamente dal plasma del cordone ombelicale umano sono in grado di promuovere l'osteogenesi e inibire l'OCgenesi in modelli murini di OP [28].

Uno dei principali svantaggi del potenziale utilizzo delle EV come agenti terapeutici è la loro breve emivita in circolo, poiché tendono ad accumularsi soprattutto in fegato e polmoni. Anche in questo caso, però, sono già oggetto di studio varie strategie risolutive, come per esempio l'aggiunta di aptameri specifici per le BMSC, che hanno permesso di ottenere ottimi risultati in vivo, riducendo la perdita di massa ossea e migliorando la rigenerazione in modelli di frattura [29]. Le EV potrebbero anche rappresentare adiuvanti di altre strategie terapeutiche, potenziandone l'efficacia. Per esempio, nella riparazione ossea, i nanotubi di titanio modificati con esosomi derivati da BMM trattati con BMP2 sono in grado di aumentare notevolmente l'angiogenesi e l'osteogenesi e potrebbero, quindi, rappresentare nuovi biomateriali [30]. Risultati simili sono stati ottenuti con scaffold di tricalciofosfato ( $\beta$-TCP) modificati con esosomi di MSC (da iPS) umane, ottenendo un netto beneficio nella riparazione ossea, principalmente attribuito all'attivazione del pathway di PI3K/Akt nelle BMSC [31]. Queste prospettive sono sicuramente appetibili e innovative; tuttavia, c'è ancora bisogno di sviluppare protocolli e linee guida standard per permettere la purificazione e l'utilizzo di EV in clinica, oltre alla difficoltà di mantenerne alta l'emivita e indirizzarle correttamente sul target. Un'ultima riflessione riguarda invece il potenziale terapeutico che vede le EV non come veicoli, ma come target esse stesse. Bloccare il rilascio delle EV osteoblastiche (via Imipramine) ricche di RANKL protegge infatti i topi dalla perdita ossea indotta da OVX [32].

Funding Note Open access funding provided by Università degli Studi di Siena within the CRUI-CARE Agreement.

Conflitto di interesse Gli autori Simone Bianciardi, Daniela Merlotti e Maria Materozzi dichiarano di non avere conflitti di interesse.

Consenso informato Lo studio presentato in questo articolo non ha richiesto sperimentazione umana.

Studi sugli animali Gli autori di questo articolo non hanno eseguito studi sugli animali.

Nota della casa editrice Springer Nature rimane neutrale in riguardo alle rivendicazioni giurisdizionali nelle mappe pubblicate e nelle affiliazioni istituzionali.

Open Access This article is licensed under a Creative Commons Attribution 4.0 International License, which permits use, sharing, adaptation, distribution and reproduction in any medium or format, as long as you give appropriate credit to the original author(s) and the source, provide a link to the Creative Commons licence, and indicate if changes were made. The images or other third party material in this article are included in the article's Creative Commons licence, unless indicated otherwise in a credit line to the material. If material is not included in the article's Creative Commons licence and your intended use is not permitted by statutory regulation or exceeds the permitted use, you will need to obtain permission directly from the copyright holder. To view a copy of this licence, visit http://creativecommons.org/licenses/by/4.0/.

\section{Bibliografia}

1. van Niel G, D'Angelo G, Raposo G (2018) Shedding light on the cell biology of extracellular vesicles. Nat Rev Mol Cell Biol 19:213-228 
2. Samanta S, Rajasingh S, Drosos N et al (2018) Exosomes: new molecular targets of diseases. Acta Pharmacol Sin 39:501-513

3. Kalluri R, LeBleu VS (2020) The biology, function, and biomedical applications of exosomes. Science 367:640

4. Gao M, Gao W, Papadimitriou JM et al (2018) Exosomes - the enigmatic regulators of bone homeostasis. Bone Res 6:36

5. Yang X, Yang J, Lei P et al (2019) LncRNA MALAT1 shuttled by bone marrow-derived mesenchymal stem cells-secreted exosomes alleviates osteoporosis through mediating microRNA-34c/SATB2 axis. Aging 11:8777-8879

6. Wei Y, Tang C, Zhang J et al (2019) Extracellular vesicles derived from the mid-to-late stage of osteoblast differentiation markedly enhance osteogenesis in vitro and in vivo. Biochem Biophys Res Commun 514:252-258

7. Cappariello A, Loftus A, Muraca M et al (2018) Osteoblastderived extracellular vesicles are biological tools for the delivery of active molecules to bone. J Bone Miner Res Off J Am Soc Bone Miner Res 33:517-533

8. Minamizaki T, Nakao Y, Irie Y et al (2020) The matrix vesicle cargo miR-125b accumulates in the bone matrix, inhibiting bone resorption in mice. Commun Biol 3:1-11

9. Yang JX, Xie P, Li YS et al (2020) Osteoclast-derived miR23a-5p-containing exosomes inhibit osteogenic differentiation by regulating Runx2. Cell Signal 70:109504

10. Huynh N, VonMoss L, Smith D et al (2016) Characterization of regulatory extracellular vesicles from osteoclasts. J Dent Res 95:673-679

11. Ma Q, Liang M, Wu Y et al (2019) Mature osteoclast-derived apoptotic bodies promote osteogenico differentiation via RANKLmediated reverse signaling. J Biol Chem 294:11240-11247

12. Qin Y, Peng Y, Zhao W et al (2017) Myostatin inhibits osteoblastic differentiation by suppressing osteocyte-derived exosomal microRNA-218: a novel mechanism in muscle-bone communication. J Biol Chem 292:11021-11033

13. Rossi M, Battafarano G, D'Agostini M et al (2018) The role of extracellular vesicles in bone metastasis. Int J MS Care 19:1136

14. Duan Y, Tan Z, Yang M et al (2019) PC-3-derived exosomes inhibit osteoclast differentiation by downregulating miR-214 and blocking NF- $\kappa$ B signaling pathway. BioMed Res Int 2019:1-8

15. Janowska-Wieczorek A, Wysoczynski M, Kijowski J et al (2005) Microvesicles derived from activated platelets induce metastasis and angiogenesis in lung cancer. Int J Cancer 113:752-760

16. Peinado H, Alečković M, Lavotshkin S et al (2012) Melanoma exosomes educate bone marrow progenitor cells toward a pro-metastatic phenotype through MET. Nat Med 18:883-891

17. Probert C, Dottorini T, Speakman A et al (2019) Communication of prostate cancer cells with bone cells via extracellular vesicle RNA; a potential mechanism of metastasis. Oncogene 38:17511763
18. Ye Y, Li SL, Ma YY et al (2017) Exosomal miR-141-3p regulates osteoblast activity to promote the osteoblastic metastasis of prostate cancer. Oncotarget 8:94834-94849

19. Xu Z, Liu X, Wang H et al (2018) Lung adenocarcinoma cellderived exosomal miR-21 facilitates osteoclastogenesis. Gene 666:116-122

20. Loftus A, Cappariello A, George C et al (2020) Extracellular vesicles from osteotropic breast cancer cells affect bone resident cells. J Bone Miner Res 35:396-412

21. Xu R, Shen X, Si Y (2018) MicroRNA-31a-5p from aging BMSCs links bone formation and resorption in the aged bone marrow microenvironment. Aging Cell 17:e12794

22. Weilner S, Schraml E, Wieser M et al (2016) Secreted microvesicular miR-31 inhibits osteogenic differentiation of mesenchymal stem cells. Aging Cell 15:744-754

23. Fulzele S, Mendhe B, Khayrullin A et al (2019) Muscle-derived miR-34a increases with age in circulating extracellular vesicles and induces senescence of bone marrow stem cells. Aging 11:1791-1803

24. Li D, Liu J, Guo B (2016) Osteoclast-derived exosomal miR-214$3 p$ inhibits osteoblastic bone formation. Nat Commun 7:10872

25. Furuta T, Miyaki S, Ishitobi H et al (2016) Stem cell-derived exosomes promote fracture healing in a mouse model. Stem Cells Transl Med 5:1620-1630

26. Zhang Y, Hao Z, Wang P et al (2019) Exosomes from human umbilical cord mesenchymal stem cells enhance fracture healing through HIF- $1 \alpha$-mediated promotion of angiogenesis in a rat model of stabilized fracture. Cell Prolif 52:e12570

27. Chen S, Tang Y, Liu Y et al (2019) Exosomes derived from miR-375-overexpressing human adipose mesenchymal stem cells promote bone regeneration. Cell Prolif 52:e12669

28. $\mathrm{Hu} \mathrm{Y,} \mathrm{Xu} \mathrm{R,} \mathrm{Chen} \mathrm{CY} \mathrm{et} \mathrm{al} \mathrm{(2019)} \mathrm{Extracellular} \mathrm{vesicles} \mathrm{from}$ human umbilical cord blood ameliorate bone loss in senile osteoporotic mice. Metabolism 95:93-101

29. Luo ZW, Li FXZ, Liu YW et al (2019) Aptamer-functionalized exosomes from bone marrow stromal cells target bone to promote bone regeneration. Nanoscale 11:20884-20892

30. Wei F, Li M, Crawford R et al (2019) Exosome-integrated titanium oxide nanotubes for targeted bone regeneration. Acta Biomater 86:480-492

31. Zhang J, Liu X, Li H et al (2016) Exosomes/tricalciumphosphate combination scaffolds can enhance bone regeneration by activating the PI3K/Akt signaling pathway. Stem Cell Res Ther 7:136

32. Deng L, Peng Y, Jiang Y et al (2017) Imipramine protects against bone loss by inhibition of osteoblast-derived microvesicles. Int $\mathrm{J}$ Mol Sci 18:1013 